\title{
Comments on: Some recent work on multivariate Gaussian Markov random fields
}

\author{
Stephan R. Sain ${ }^{1} \cdot$ Reinhard Furrer ${ }^{2,3}$ (D \\ Published online: 12 September 2018 \\ c) Sociedad de Estadística e Investigación Operativa 2018
}

In Some recent work on multivariate Gaussian Markov random fields, author Ying C. MacNab unifies several lines of research focused on multivariate formulations of Gaussian Markov random field (MRF) models through a coregionalization framework. MRFs are natural models for data on regular or irregular lattices, and, as the author has noted, they have found application in a wide range of scientific areas. There are significant computational advantages to MRFs that allow consideration of much larger datasets, but there are also a number of other challenges that arise with such models. The author specifically addresses two of these, namely the entanglement of spatial and non-spatial components and the enforcement for positivity condition.

Multivariate Gaussian MRFs involve the specification of a precision matrix that encompasses both the dependence between variables and the spatial dependence across location. This precision matrix is often sparse, which enables the use of sparse matrix methods to improve computational performance. However, these precision matrices have very complex structure and parameterizations, which leads to the noted issues with entanglement of the spatial and non-spatial components and difficulty with ensuring these matrices are positive definite. While the paper yields a great deal of information about these issues, there are some practical concerns for those interested in using such models that are also important. We comment on those in the following section. Next, against a statement made in the paper, the model of Sain et al. (2011) does allow a separable structure as we show.

This comment refers to the invited paper available at https://doi.org/10.1007/s11749-018-0605-3.

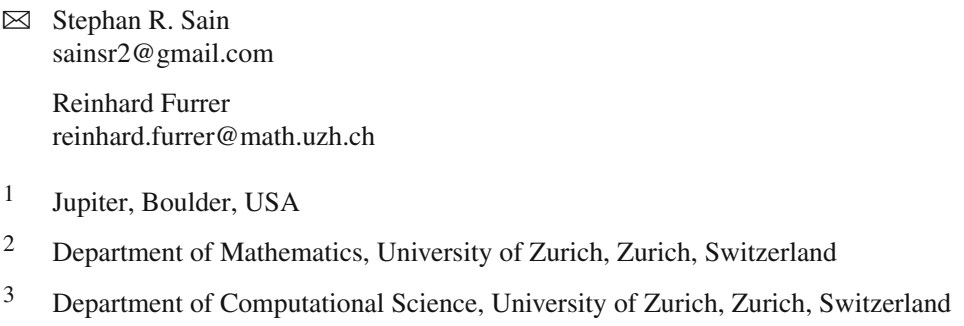




\section{Practical issues}

Much of the paper involves unifying the different types of multivariate MRFs through coregionalization, and a number of different model types are presented. This is an impressive undertaking and yields some interesting insights with respect to the entanglement of the spatial and non-spatial components. However, it seems that there is still not one unified model formulation that allows movement between the different approaches through some set of parameters. Thus, an analyst is stuck with choosing one model and will often not try different alternatives. Or, the analyst is tasked with implementing a number of different formulations and then trying to choose objectively in a manner similar to what was done in the examples in the paper. It is still important to provide guidance to analysts with the practical implications of the different models and, in particular, encourage the analyst to seek out different options. Of course, it remains an active research area to develop objective procedures to choose between competing models.

One important reason to consider using MRFs is the ability to capitalize on sparse matrix methods, particularly for larger datasets. The two examples discussed are useful illustrations, but they have less than 100 locations each, which, for many applications, particularly in climate and weather, remote sensing, etc., is really quite small. If the ability to use sparse methods is lost, then it is a serious drawback to this approach. While Section 6 of the paper deals with some of the computational issues, it seems that the focus was on enforcing positivity of the precision matrix. While computation for fairly small sample sizes such as those in the examples does not really need sparse methods, computational approaches that are able to handle larger datasets are critically important.

Another key issue with MRF models is that they do not, in general, lead to stationary models. More importantly, this is often due to the configuration of the lattice, which is often completely unrelated to the underlying physical process. Further, different model formulations lead to different nonstationarities. Sain and Furrer (2010) used a Kronecker product of two univariate stationary MRFs for a regular (rectangular) lattice. While stationary and very computationally efficient for larger sample sizes, the resulting covariance function has some less than desirable properties. It would be interesting to see if these different types of coregionalization models are stationary and how they compare with each other in this respect.

The paper also presents only first-order neighborhood models. For smoother fields or when approximating Gaussian random fields with a Gaussian MRF, higher-order neighborhood structures are needed (Rue and Tjelmeland 2002). Such neighborhood structures are conceptually similar for the different models presented in the paper, but we presume that they lead to different practical implications. Different neighborhood structures add another level of complexity to what are already fairly complex statistical models. However, it would be useful to see whether such models fit into a ready-to-use Bayesian software environment. Further, a discussion of likelihood estimation would also be useful to help address issues such as choice of parameterization or the impact of transformations or constraints for parameters to improve estimation. 


\section{Separability}

The author notes in Section 3.2 that, "The Sain et al. (2011) framework does not contain separable models in its family of MGMRFs." This is not generally the case. Consider the bivariate example outlined in the Sain et al. (2011) paper with dependency connections defined to capture: (1) dependence between variables at a particular location, (2) spatial dependence within a variable, and (3) cross-spatial dependence. After some simplification, Eq. (5) of Sain et al. (2011) is written as

$$
\left[I_{n} \otimes \tau^{1 / 2}\right]\left[I_{n} \otimes A-\left[\begin{array}{ccc}
0 & & B \delta_{i j} \\
& \ddots & \\
B^{\prime} \delta_{i j} & & 0
\end{array}\right]\right]^{-1}\left[I \otimes \tau^{1 / 2}\right],
$$

where $\tau=\left[\tau_{1}, \ldots, \tau_{p}\right]^{\prime}$ is a $p$-vector of scale parameters where $p$ is the multivariate dimension, $A$ is a $p \times p$ symmetric matrix with 1 s on the diagonal and representing the "within-location" dependence structure, $B$ is a $p \times p$ matrix of spatial dependence parameters, and $\delta_{i j}$ representing neighborhood structure (i.e., $\delta_{i j}=1$ location $i$ and location $j$ are neighbors and $\delta_{i j}=0$ otherwise). Further restricting $B$ to be symmetric (and removing potential for asymmetric cross-dependence) and setting $B=\phi A$ where $\phi$ can now be thought of as a general spatial dependence parameter, Eq. (5) from Sain et al. (2011) can be simplified to

$$
\left[I_{n} \otimes \tau^{1 / 2}\right]\left[I_{n} \otimes A-\phi\left[\begin{array}{ccc}
0 & & A \delta_{i j} \\
& \ddots & \\
A \delta_{i j} & & 0
\end{array}\right]\right]^{-1}\left[I \otimes \tau^{1 / 2}\right] .
$$

With some rearranging, this becomes $\left[I_{n} \otimes \tau^{1 / 2}\right]\left[\left(I_{n}-\phi H\right) \otimes A\right]^{-1}\left[I \otimes \tau^{1 / 2}\right]$, where $H$ is an $n \times n$ matrix with diagonal elements $h_{i i}=0$ and off-diagonal elements $h_{i j}=\delta_{i j}$. This gives the necessary Kronecker form, effectively separating the spatial component $\left(I_{n}-\phi H\right)$ and the non-spatial component $(A)$.

The Sain et al. (2011) framework requires the specification of connections both within and across the lattices associated with the different variables. It also requires different assumptions and constraints on what values the parameters associated with those connections can take on. Together, these two steps are ultimately controlled by the modeler or analyst and specify the model structure, and it is up to the modeler or analyst to carefully consider these two steps to span the desired model types. There are different model structures under this formulation that would have a separable form [such as the example discussed in Sain et al. (2011) and its extension to more than two variables], and there are lattice structures and different specifications of connections (or no connections, which is equivalent to forcing certain parameters to have a zero value) that can easily be seen to not have a separable form. 


\section{References}

Rue H, Tjelmeland H (2002) Fitting Gaussian Markov random fields to Gaussian field. Scand J Stat 29:31-49 Sain SR, Furrer R (2010) Combining climate model output via model correlations. Stoch Environ Res Risk Assess 24(6):821-829

Sain SR, Furrer R, Cressie N (2011) A spatial analysis of multivariate output from regional climate models. Ann Appl Stat 5(1):150-175 